\title{
GORD
}

\section{Body mass index and chronic unexplained gastrointestinal symptoms: an adult endoscopic population based study}

\author{
P Aro, J Ronkainen, N J Talley, T Storskrubb, E Bolling-Sternevald, L Agréus
}

Gut 2005;54:1377-1383. doi: 10.1136/gut.2004.057497

See end of article for authors' affiliations

Correspondence to:

Correspondence to:

Clinic College of Medicine,

Center for Enteric

Neurosciences

Translational and

Epidemiological Research

(CENTER), Mayo Clinic,

200 First St, PL-6-56,

Rochester, MN 55905,

USA; talley.nicholas@

mayo.edu

Revised version received 14 April 2005

Accepted for publication

10 May 2005

Published online first

25 May 2005
Background: We aimed to determine whether obese subjects experience more gastro-oesophageal reflux (GORS) symptoms than normal subjects, and further to determine if this association was explained by oesophagitis or medications that lower oesophageal sphincter pressure.

Methods: In a representative Swedish population, a random sample $(n=1001$, mean age 53.5 years, $51 \%$ women) had upper endoscopy. GORS was defined as any bothersome heartburn or acid regurgitation.

Results: The prevalence of obesity (body mass index $\geqslant 30$ ) was $16 \%$; oesophagitis was significantly more prevalent in obesity (26.5\%) than in normal subjects (9.3\%). There were associations between obesity and GORS (odds ratio (OR) 2.05 (95\% confidence interval (Cl) 1.39, 3.01)), epigastric pain (OR $1.63(95 \% \mathrm{Cl}$ 1.05, 2.55)), irritable bowel symptoms (OR $1.58(95 \% \mathrm{Cl} 1.05,2.38))$, any abdominal pain (OR 1.59 $(95 \% \mathrm{Cl} 1.08,2.35))$, vomiting (OR 3.11 (95\% Cl 1.18, 8.20)), retching (OR $1.74(95 \% \mathrm{Cl} 1.1 .3,2.67))$, diarrhoea (OR $2.2(95 \% \mathrm{Cl} 1.38,3.46))$, any stool urgency (OR $1.60(95 \% \mathrm{Cl} 1.04,2.47))$, nocturnal urgency (OR $2.57(95 \% \mathrm{Cl} 1.33,4.98))$, and incomplete rectal evacuation (OR $1.64(95 \% \mathrm{Cl} 1.09,2.47))$, adjusting for age, sex, and education. When subjects with oesophagitis and peptic ulcer were excluded, only diarrhoea, incomplete evacuation, and vomiting were significantly associated with obesity. The association between GORS and obesity remained significant adjusting for medication use (OR $1.9195 \% \mathrm{Cl}$ $1.3,3.0) 1$.

Conclusions: GORS is associated with obesity; this appears to be explained by increased upper endoscopy findings in obesity.
7 astro-oesophageal reflux symptoms (GORS) are highly prevalent in Western nations, ${ }^{1-5}$ and may be increasing in incidence in Asia for unknown reasons. ${ }^{67}$ Similarly, the development of obesity has reached epidemic proportions in the Western world, which also remains largely unexplained. ${ }^{89}$ Obesity is important because it induces a major psychological burden ${ }^{10}$ and has a substantial impact on morbidity and quality of life. ${ }^{11}{ }^{12}$

We have previously reported that body mass index (BMI) appeared to be an independent risk factor for the presence of heartburn and acid regurgitation in a community based population study in the USA. ${ }^{13}$ Others have observed similar associations in Sweden but there have also been contradictory reports. ${ }^{14} 15$ We have also observed an association between obesity and symptoms of diarrhoea in population based studies from the USA, Australia, and in a New Zealand birth cohort of young adults. ${ }^{16-18}$ However, these studies were all in uninvestigated subjects, and the relationship between BMI and unexplained upper and lower gastrointestinal symptoms remains to be clarified.

A number of drugs have been reported to lower oesophageal sphincter pressure ${ }^{19-23}$ and an association between the use of such drugs and an increased risk of oesophageal adenocarcinoma has also been observed. ${ }^{24}$ However, population based studies that include endoscopic data have not investigated how important medications are in causing oesophagitis, and whether their use mediates the possible association between BMI and GORS.

In this study, we aimed to evaluate the relationship between measured BMI and specific gastrointestinal symptoms in a community based population that was being evaluated by oesophagogastroduodenoscopy. We hypothe- sised that the association of obesity with symptoms of GORS would be largely explained by underlying oesophagitis or by medications that could potentially aggravate gastro-oesophageal reflux.

\section{MATERIALS AND METHODS Setting}

The setting consisted of two neighbouring communities in Northern Sweden, Kalix and Haparanda, with 18408 and 10580 inhabitants (as of December 1998); 78\% lived in city populated areas during the year 2000 compared with the Swedish national average of $84 \%$. The distribution of age and sex was similar to the national average in Sweden in both communities, although unemployment status, income, and the proportion with a higher education were slightly lower.

\section{Sampling}

Using the computerised national population register, covering all citizens in the two communities by date of birth order, a representative sample was generated. Every seventh adult $(n=3000)$ from the target population $(20-80$ years of age, $\mathrm{n}=21610$ in September 1998) was drawn, a procedure equivalent to random sampling. The sampled subjects were then, by a computerised process, given an identity number (ID 1-3000) in random order.

Abbreviations: $A S Q$, abdominal symptom questionnaire; $B M I$, body mass index; OEG, oesophagogastroduodenoscopy; GORD, gastrooesophageal reflux disease; GORS, gastro-oesophageal reflux symptoms; ID, identity number; IBS, irritable bowel syndrome; LOS, lower oesophageal sphincter; $O R$, odds ratio 


\section{Study design and logistics}

The study population $(\mathrm{n}=3000)$ was contacted by mail and invited to take part; this invitation included a validated questionnaire, the abdominal symptom questionnaire (ASQ) (see below) to be returned by mail. Up to two remainders were applied when necessary; 140 subjects were unavailable at the time of invitation (21 dead; 38 migrated or questionnaire returned by relatives; 17 mentally retarded or having dementia; and 76 for other reasons). Thus 2860 of the original study population were eligible for inclusion.

Responders were invited to a visit in the clinic in ID order, starting with the lowest available ID. Subjects reported the absence/presence of gastrointestinal symptoms using the ASQ questionnaire at the visit, as described below. The study population was divided into five parts in ascending order for logistic reasons, ID 1-600, 601-1200, and so forth, and the first subset of study subjects was approached with the mailed ASQ questionnaire in December 1998. The study was approved by the Umeå University ethics committee and conducted in accordance with the revised Declaration of Helsinki.

\section{Assessments}

Abdominal symptom questionnaire (ASQ)

This self-administered questionnaire assesses symptoms from the upper and lower part of the abdomen and has been validated in Sweden. ${ }^{25} 26$ A standardised procedure for the administration of the questionnaire at the visit was used. The ASQ includes questions describing the presence or absence (yes/no) of 27 troublesome gastrointestinal symptoms over the preceding three months. In order to better reflect the Rome I definitions of functional gastrointestinal disorders, ${ }^{27}$ three questions were added to the present version. ${ }^{28}$ All participants were also asked if they had been troubled by any of 11 listed descriptors of abdominal pain, ${ }^{26}$ in addition to symptom location (upper, centre, or lower abdominal, right and left flank, respectively).

\section{Demographics and history}

Demographic data were collected at the clinic visit (sex, age, weight, tobacco use, and language). The subject's level of education and number of inhabitants in their household was confirmed by questions in the ASQ at the visit to the clinic.

\section{Definitions of symptom groups}

Subjects were classified according to their symptom patterns as defined below:

\section{(1) Gastro-oesophageal reflux symptoms (GORS)}

GORS were defined as the presence of any troublesome heartburn and/or acid regurgitation over the past three months. ${ }^{29} 30$

\section{(2) Dyspepsia}

Dyspepsia was defined as any troublesome pain or discomfort expressed as one or more of the 11 listed pain modalities located in the upper (epigastric) part of the abdomen, and/or nausea, early satiety, or uncomfortable feeling of fullness after a meal. This is consistent with the Rome II definition (except for upper abdominal bloating which was not asked about in the ASQ). ${ }^{26}$

(3) Irritable bowel syndrome (IBS)

IBS was defined as any of the troublesome abdominal pain modalities located at any site plus concomitant bowel habit disturbances (constipation, diarrhoea, or alternating constipation and diarrhoea). ${ }^{28}$ This simple definition has been used previously and shown to produce results reasonably concordant with the Rome criteria in Sweden. ${ }^{28}$
(4) Epigastric pain or discomfort

Epigastric pain in the ASQ was defined as troublesome pain or discomfort expressed as one or more of the 11 listed pain or discomfort modalities indicated in the epigastric part of the abdomen only. This definition is based on the Rome I definition of dyspepsia.

\section{(5) Abdominal pain}

Abdominal pain was defined as troublesome pain or discomfort expressed as one or more of the 11 listed pain or discomfort modalities indicated anywhere in the abdomen.

\section{Response rate}

A total of 2122 individuals completed the postal questionnaire, which corresponds to a response rate of $74.2 \%$ after two postal reminders. These responders were representative of the local population. ${ }^{31}$ In order to complete the 1001 upper endoscopies, 1563 responders to the ASQ were approached; 364 declined, 74 had moved or could not be reached, and 124 had medical contraindications. Thus the response rate for those eligible for investigation was $73.3 \%$. Sex and age distribution for the 1001 subjects (488 males $(48.8 \%)$ ) who responded to the questionnaire at both assessments (mean age 54 years) closely reflect the pattern in the Swedish population. ${ }^{31}$ The study subjects who refused endoscopy were very similar demographically to the 1001 subjects evaluated (data not shown). Hence a representative cohort of 1001 invited for upper endoscopy was evaluated. Of the subjects endoscoped, 10 did not have BMI data collected, leaving 991 for analysis.

Data on the prevalence of endoscopic findings in this population are presented elsewhere. ${ }^{32}$ Oesophagitis was classified according to the Los Angeles classification system; detailed data on oesophagitis and its associations with GORS are published elsewhere. ${ }^{33}$

\section{Oesophagogastroduodenoscopy (OEG)}

Upper endoscopies were performed by both primary and secondary care physicians in the two clinics who provided sole medical cover in the area. The endoscopists were unaware of the symptoms of the subjects before and during endoscopy. ${ }^{34}$

\section{Body mass index categories}

Height and weight were measured at the endoscopy visit. Data on weight and height were used to calculate BMI $(\mathrm{kg} /$ $\mathrm{m}^{2}$ ). Participants were categorised based on BMI as underweight (BMI $<18.5$ ), normal (BMI $\geqslant 18.5$ and $<25$ ), overweight (BMI $\geqslant 25$ and $<30$ ), obese class I (BMI $\geqslant 30$ and $<35)$, class II ( $\geqslant 35$ and $<40)$, and class III $(\geqslant 40) \cdot{ }^{35}$ Because there were relatively few subjects in the extreme obesity categories, these were all combined.

\section{Medications}

Data on medication use were recorded after endoscopy. In addition to any acid suppressing drug, medications that were concurrently being taken that may reduce lower oesophageal sphincter (LOS) pressure (nitrates, theophylline, calcium channel blockers, opiates, beta agonists, phenothiazines, tricyclic antidepressive drugs, nicotine substitutes, anticholinergics, and benzodiazepines) were recorded. ${ }^{19-24}$

\section{Statistical analysis}

Prevalence is shown as percentage with $95 \%$ confidence interval (CI). We used a logistic regression analysis to assess the association between the presence of each specific gastrointestinal symptom (the binary dependent variable) and BMI (entered as a categorised independent variable), adjusting for age, sex, and education use. The odds ratios 
Table 1 Distribution of demographic variables by body mass index (BMI) categories

\begin{tabular}{|c|c|c|c|c|c|c|c|c|c|c|c|}
\hline \multirow[b]{3}{*}{ BMI category } & \multirow{3}{*}{$\begin{array}{l}\text { n } \\
\% \\
(95 \% \mathrm{Cl})\end{array}$} & \multicolumn{2}{|l|}{ Age } & \multicolumn{2}{|l|}{ Sex } & \multicolumn{2}{|l|}{ Education } & \multicolumn{2}{|l|}{ Smokingt } & \multicolumn{2}{|c|}{ Alcohol/week } \\
\hline & & $\leqslant 54^{*}$ & $>54^{*}$ & & & & & & & & \\
\hline & & $n \%$ & $n \%$ & Female $^{*}$ & Male* $^{*}$ & Low* & High* & No* & Yes* & $\leqslant 100 \mathrm{~g}^{*}$ & $>100 \mathrm{~g}^{*}$ \\
\hline \multirow{2}{*}{$\begin{array}{l}\text { Underweight } \\
\qquad(<18.5)\end{array}$} & 8 & 4 & 4 & 6 & 2 & 5 & 3 & 6 & 2 & 8 & 0 \\
\hline & $\begin{array}{l}0.8 \\
(0.3-1.4)\end{array}$ & $\begin{array}{l}0.8 \\
(0.0-1.6)\end{array}$ & $\begin{array}{l}0.8 \\
(0.0-1.6)\end{array}$ & $\begin{array}{l}1.2 \\
(0.3-2.1)\end{array}$ & $\begin{array}{l}0.4 \\
(0.0-1.0)\end{array}$ & $\begin{array}{l}0.9 \\
(0.1-1.7)\end{array}$ & $\begin{array}{l}0.7 \\
(0.0-1.5)\end{array}$ & $\begin{array}{l}0.7 \\
(0.1-1.3)\end{array}$ & $\begin{array}{l}1.1 \\
(0.0-2.6)\end{array}$ & $\begin{array}{l}0.9 \\
(0.3-1.5)\end{array}$ & \\
\hline \multirow{3}{*}{$\begin{array}{l}\text { Normal weight } \\
\qquad(\geqslant 18.5-<25)\end{array}$} & 365 & 200 & 165 & 213 & 152 & 177 & 183 & 280 & 85 & 331 & 34 \\
\hline & 36.8 & 41.1 & 32.7 & 41.9 & 31.5 & 31.2 & 45.0 & 34.8 & 45.7 & 37.5 & 31.2 \\
\hline & $(33.8-39.8)$ & $(36.7-45.5)$ & $(28.6-36.8)$ & $(37.6-46.2)$ & $(27.4-35.6)$ & $(27.4-35.0)$ & $(40.2-49.8)$ & $(31.5-38.1)$ & $(38.5-52.9)$ & $(34.3-40.7)$ & $(22.5-39.9)$ \\
\hline \multirow{3}{*}{$\begin{array}{l}\text { Overweight } \\
\qquad(\geqslant 25-<30)\end{array}$} & 456 & 211 & 245 & 197 & 259 & 281 & 166 & 384 & 72 & 399 & 57 \\
\hline & 46.0 & 43.3 & 48.6 & 38.8 & 53.6 & 49.5 & 40.9 & 47.7 & 38.7 & 45.2 & 52.3 \\
\hline & $(42.9-49.1)$ & ) (38.9-47.7) & $(44.2-53.0)$ & ) $(34.6-43.0)$ & ) $(49.2-58.0)$ & ) (45.4-53.6) & ) (36.1-45.7) & $(44.2-51.2)$ & ) $(31.7-45.7)$ & $(41.9-48.5)$ & ) (42.9-61.7) \\
\hline \multirow{3}{*}{$\begin{array}{l}\text { Obese } \\
\qquad(\geqslant 30)\end{array}$} & 162 & 72 & 90 & 92 & 70 & 105 & 55 & 135 & 27 & 144 & 18 \\
\hline & 16.3 & 14.9 & 17.9 & 18.1 & 14.6 & 18.5 & 13.5 & 16.8 & 14.5 & 16.3 & 16.5 \\
\hline & (14.0-18.7) & ) (11.7-18.1) & $(14.6-21.2)$ & ) (14.8-21.4) & (11.5-17.7) & ) (14.9-21.3) & ) (10.2-16.8) & $(14.2-19.4)$ & 1) $(9.4-19.6)$ & (13.9-18.7) & $(9.5-23.5)$ \\
\hline Total & 991 & 487 & 504 & 508 & 483 & 568 & 407 & 805 & 186 & 882 & 109 \\
\hline
\end{tabular}

(OR) for a given specific symptom and 95\% CI were computed from the coefficients (and standard errors) in the logistic regression models in which BMI was categorised as described above. Individual gastrointestinal symptoms, groups of gastrointestinal symptoms, and other possible exposure variables were analysed separately in different analyses by endoscopy findings. Multiple logistic regression was used to assess the association between BMI and GORS or separately oesophagitis, adjusting for medication use as well as age, sex, and education level. Linear regression analysis was applied to analyse the independent associations between BMI and possible exposure variables.

\section{RESULTS}

\section{Prevalence of obesity}

The prevalence of those underweight was $0.8 \%(n=8)$; these subjects were excluded leaving 983 subjects in the subsequent analyses. The prevalence of being overweight was $46 \%$ $(\mathrm{n}=456(95 \%$ CI $42.9,49.1))$ while the prevalence of obesity was $16 \%(n=162(95 \%$ CI $14.0,18.7))$. Table 1 shows the proportion of patients in each BMI category, as a whole and by gender, age groups, education levels, smoking and alcohol status. Smoking was independently associated with decreased BMI by linear regression analysis (beta coefficient -0.7) and low education was associated with increased BMI (beta coefficient 0.6); alcohol use was not significant.

\section{Prevalence of troublesome gastrointestinal} complaints and upper endoscopy findings

At the time of endoscopy, $65.6 \%$ of 1001 subjects reported one or more troublesome gastrointestinal complaints on the questionnaire completed prior to endoscopy. The prevalence of major endoscopic findings by BMI category is summarised in table 2. Of those with oesophagitis $(n=155)$, most were grade $\mathrm{A}(\mathrm{n}=109)$; 39 had grade $\mathrm{B}$, three grade $\mathrm{C}$, two grade $\mathrm{D}$, and two were unable to be classified. There were more endoscopic findings in obese subjects than in normal weight subjects, and the differences were significant for oesophagitis and gastric ulcer; the prevalence of oesophagitis in obesity was $26.5 \%$ (95\% CI 19.7, 33.3) versus $9.3 \%$ (95\% CI 6.3, 12.3 ) in normal weight subjects while the prevalence of gastric ulcer in obesity was $5.6 \%$ (95\% CI 2.0, 9.1) versus $1.4 \%$ (95\% CI $0.2,2.6$ ) in normal weight subjects.

\section{Relationship between BMI, gastrointestinal symptoms, and other exposure factors}

In the total cohort, the distribution of individual gastrointestinal symptoms by BMI categories is summarised in table 3.

There were significant associations between obesity and GORS (OR 2.05 (95\% CI 1.39, 3.01)), epigastric pain (OR 1.63 (95\% CI 1.05, 2.55)), IBS (OR 1.58 (95\% CI 1.05, 2.38)), any abdominal pain (OR 1.59 (95\% CI $1.08,2.35)$ ), vomiting (OR 3.11 (95\% CI 1.18, 8.20)), retching (OR 1.74 (95\% CI 1.1.3, $2.67)$ ), diarrhoea (OR $2.21(95 \%$ CI $1.38,3.46))$, any stool urgency (OR 1.60 (95\% CI 1.04, 2.47)), nocturnal urgency (OR 2.57 (95\% CI 1.33, 4.98)), and feelings of incomplete rectal evacuation (OR $1.64(95 \%$ CI $1.09,2.47)$ ), adjusting for age, sex, and education (table 4).

When subjects with oesophagitis, peptic ulcer, and cancer at endoscopy were excluded, diarrhoea (OR 1.94 (95\% CI $1.13,3.32$ )), feelings of incomplete rectal evacuation (OR 1.68

Table 2 Prevalence (\%) of peptic ulcer disease, oesophagitis, and gastric cancer in different body mass index (BMI) categories

\begin{tabular}{|c|c|c|c|c|}
\hline \multirow[b]{2}{*}{ Endoscopic finding } & \multicolumn{4}{|c|}{ BMI category (n (\%) [95\% CI]) } \\
\hline & $\begin{array}{l}\text { Underweight } \\
(\text { BMI }<18.5) \\
(n=8)\end{array}$ & $\begin{array}{l}\text { Normal } \\
(B M I \geqslant 18.5<25) \\
(n=365)\end{array}$ & $\begin{array}{l}\text { Overweight } \\
(B M I \geqslant 25<30) \\
(n=456)\end{array}$ & $\begin{array}{l}\text { Obese } \\
(B M I \geqslant 30) \\
(n=162)\end{array}$ \\
\hline Gastric ulcer & $0(0)$ & $\begin{array}{l}5(1.4) \\
{[0.2-2.6]}\end{array}$ & $\begin{array}{l}6(1.3) \\
{[0.3-2.4]}\end{array}$ & $\begin{array}{l}9(5.6) \\
{[2.0-9.1]}\end{array}$ \\
\hline Duodenal ulcer & $0(0)$ & $\begin{array}{l}7(1.9) \\
{[0.5-3.3]}\end{array}$ & $\begin{array}{l}9(2.0) \\
{[0.7-3.2]}\end{array}$ & $\begin{array}{l}4(2.5) \\
{[0.1-4.9]}\end{array}$ \\
\hline Oesophagitis & $\begin{array}{l}1(12.5) \\
{[10.4-35.4]}\end{array}$ & $\begin{array}{l}34(9.3) \\
{[6.3-12.3]}\end{array}$ & $\begin{array}{l}76(16.7) \\
{[13.2-20.1]}\end{array}$ & $\begin{array}{l}43(26.5) \\
{[19.7-33.3]}\end{array}$ \\
\hline Cancer & $0(0)$ & $0(0)$ & $\begin{array}{l}1(0.2) \\
{[0.0-0.7]}\end{array}$ & $0(0)$ \\
\hline
\end{tabular}


Table 3 Distribution of gastrointestinal symptoms by body mass index (BMI) categories

\begin{tabular}{|c|c|c|c|}
\hline Gastrointestinal symptom & $\begin{array}{l}\text { Normal weight } \\
\text { (BMI }<25) \\
\text { n (\% of category) } \\
{[95 \% \mathrm{Cl}]}\end{array}$ & $\begin{array}{l}\text { Overweight } \\
\text { (BMI } \geqslant 25-<30) \\
\text { n }(\% \text { of category) } \\
{[95 \% \mathrm{Cl}]}\end{array}$ & $\begin{array}{l}\text { Obese } \\
(\mathrm{BMI} \geqslant 30) \\
n(\% \text { of category }) \\
{[95 \% \mathrm{Cl}]}\end{array}$ \\
\hline Weight loss & $\begin{array}{l}16(4.3) \\
{[2.3-6.4]}\end{array}$ & $\begin{array}{l}5(1.1) \\
{[0.1-2.1]}\end{array}$ & $\begin{array}{l}1(0.6) \\
{[0.0-1.8]}\end{array}$ \\
\hline Loss of appetite (anorexia) & $\begin{array}{l}15(4.0) \\
{[2.0-6.0]}\end{array}$ & $\begin{array}{l}18(4.0) \\
{[2.2-5.8]}\end{array}$ & $\begin{array}{l}3(1.9) \\
{[0.0-3.9]}\end{array}$ \\
\hline Uncomfortable feeling of fullness & $\begin{array}{l}61(16.4) \\
{[12.7-20.2]}\end{array}$ & $\begin{array}{l}82(18.2) \\
{[14.7-21.8]}\end{array}$ & $\begin{array}{l}29(18.4) \\
{[12.3-24.4]}\end{array}$ \\
\hline Difficulty swallowing & $\begin{array}{l}23(6.2) \\
{[3.7-8.6]}\end{array}$ & $\begin{array}{l}28(6.2) \\
{[4.0-8.4]}\end{array}$ & $\begin{array}{l}16(9.9) \\
{[5.3-14.6]}\end{array}$ \\
\hline Retching & $\begin{array}{l}78(21.0) \\
{[16.9-25.2]}\end{array}$ & $\begin{array}{l}103(22.7) \\
{[18.9-26.6]}\end{array}$ & $\begin{array}{l}53(32.7) \\
{[25.5-39.9]}\end{array}$ \\
\hline Acid regurgitation & $\begin{array}{l}80(21.6) \\
{[17.4-25.7]}\end{array}$ & $\begin{array}{l}115(25.5) \\
{[21.5-29.5]}\end{array}$ & $\begin{array}{l}62(38.3) \\
{[30.8-45.8]}\end{array}$ \\
\hline Early satiation & $\begin{array}{l}45(12.1) \\
{[8.8-15.4]}\end{array}$ & $\begin{array}{l}63(13.9) \\
{[10.7-17.1]}\end{array}$ & $\begin{array}{l}19(11.7) \\
{[6.8-16.7]}\end{array}$ \\
\hline Nausea & $\begin{array}{l}49(13.2) \\
{[9.7-16.6]}\end{array}$ & $\begin{array}{l}59(13.0) \\
{[9.9-16.1]}\end{array}$ & $\begin{array}{l}25(15.4) \\
{[9.9-21.0]}\end{array}$ \\
\hline Vomiting & $\begin{array}{l}9(2.4) \\
{[0.9-4.0]}\end{array}$ & $\begin{array}{l}14(3.1) \\
{[1.5-4.7]}\end{array}$ & $\begin{array}{l}10(6.2) \\
{[2.5-9.9]}\end{array}$ \\
\hline Heartburn & $\begin{array}{l}100(26.9) \\
{[22.4-31.4]}\end{array}$ & $\begin{array}{l}159(35.1) \\
{[30.7-39.5]}\end{array}$ & $\begin{array}{l}68(42.5) \\
{[34.8-50.2]}\end{array}$ \\
\hline Central chest pain & $\begin{array}{l}71(19.2) \\
{[15.2-23.2]}\end{array}$ & $\begin{array}{l}98(22.0) \\
{[18.1-25.8]}\end{array}$ & $\begin{array}{l}42(26.1) \\
{[19.3-32.9]}\end{array}$ \\
\hline Burning feeling rising in chest & $\begin{array}{l}53(14.4) \\
{[10.8-18.0]}\end{array}$ & $\begin{array}{l}89(19.9) \\
{[16.2-23.6]}\end{array}$ & $\begin{array}{l}39(24.7) \\
{[18.0-31.4]}\end{array}$ \\
\hline Constipation & $\begin{array}{l}96(25.9) \\
{[21.4-30.3]}\end{array}$ & $\begin{array}{l}96(21.2) \\
{[17.5-25.0]}\end{array}$ & $\begin{array}{l}38(23.8) \\
{[17.2-30.3]}\end{array}$ \\
\hline Diarrhoea & $\begin{array}{l}64(19.9) \\
{[15.6-24.3]}\end{array}$ & $\begin{array}{l}106(26.1) \\
{[21.8-30.4]}\end{array}$ & $\begin{array}{l}46(33.1) \\
{[25.3-40.9]}\end{array}$ \\
\hline Alternating constipation/diarrhoea & $\begin{array}{l}45(12.3) \\
{[8.9-15.7]}\end{array}$ & $\begin{array}{l}58(13.0) \\
{[9.8-16.1]}\end{array}$ & $\begin{array}{l}23(14.5) \\
{[9.0-19.9]}\end{array}$ \\
\hline Feeling incomplete rectal evacuation & $\begin{array}{l}100(27.3) \\
{[22.8-31.9]}\end{array}$ & $\begin{array}{l}125(27.8) \\
{[23.7-32.0]}\end{array}$ & $\begin{array}{l}60(37.7) \\
{[30.2-45.3]}\end{array}$ \\
\hline Pain at defecation & $\begin{array}{l}43(11.7) \\
{[8.4-15.0]}\end{array}$ & $\begin{array}{l}43(9.5) \\
{[6.8-12.2]}\end{array}$ & $\begin{array}{l}16(10.0) \\
{[5.4-14.6]}\end{array}$ \\
\hline Pain relieved by defecation & $\begin{array}{l}75(20.4) \\
{[16.3-24.6]}\end{array}$ & $\begin{array}{l}96(21.2) \\
{[17.4-25.0]}\end{array}$ & $\begin{array}{l}33(20.5) \\
{[14.3-26.7]}\end{array}$ \\
\hline Straining & $\begin{array}{l}96(25.9) \\
{[21.4-30.3]}\end{array}$ & $\begin{array}{l}103(22.8) \\
{[19.0-26.7]}\end{array}$ & $\begin{array}{l}40(24.7) \\
{[18.0-31.3]}\end{array}$ \\
\hline Urgency & $\begin{array}{l}73(19.8) \\
{[15.7-23.8]}\end{array}$ & $\begin{array}{l}94(20.8) \\
{[17.1-24.5]}\end{array}$ & $\begin{array}{l}46(28.6) \\
{[21.6-35.5]}\end{array}$ \\
\hline Flatus & $\begin{array}{l}82(22.2) \\
{[18.0-26.5]}\end{array}$ & $\begin{array}{l}130(28.6) \\
{[24.4-32.7]}\end{array}$ & $\begin{array}{l}46(28.8) \\
{[21.7-35.8]}\end{array}$ \\
\hline Borborygmi & $\begin{array}{l}106(28.7) \\
{[24.0-33.3]}\end{array}$ & $\begin{array}{l}140(30.9) \\
{[26.7-35.2]}\end{array}$ & $\begin{array}{l}43(27.0) \\
{[22.3-36.5]}\end{array}$ \\
\hline Abdominal distension & $\begin{array}{l}133(36.2) \\
{[31.3-41.2]}\end{array}$ & $\begin{array}{l}152(33.5) \\
{[29.1-37.8]}\end{array}$ & $\begin{array}{l}55(34.2) \\
{[26.8-41.5]}\end{array}$ \\
\hline Nightly urge to defecate & $\begin{array}{l}20(5.4) \\
{[3.1-7.7]}\end{array}$ & $\begin{array}{l}25(5.5) \\
{[3.4-7.6]}\end{array}$ & $\begin{array}{l}20(12.4) \\
{[7.3-17.4]}\end{array}$ \\
\hline Black stools & $\begin{array}{l}8(2.2) \\
{[0.7-3.6]}\end{array}$ & $\begin{array}{l}9(2.0) \\
{[0.7-3.3]}\end{array}$ & $\begin{array}{l}1(0.6) \\
{[0.0-1.8]}\end{array}$ \\
\hline Blood in stool & $\begin{array}{l}24(6.5) \\
{[4.0-9.0]}\end{array}$ & $\begin{array}{l}37(8.2) \\
{[5.7-10.7]}\end{array}$ & $\begin{array}{l}10(6.2) \\
{[2.5-9.9]}\end{array}$ \\
\hline Mucus & $\begin{array}{l}37(10.0) \\
{[6.9-13.1]}\end{array}$ & $\begin{array}{l}27(6.0) \\
{[3.8-8.2]}\end{array}$ & $\begin{array}{l}20(12.4) \\
{[7.3-17.4]}\end{array}$ \\
\hline
\end{tabular}

(95\% CI 1.04, 2.71)), and vomiting (OR 3.98 (95\% CI 1.26, 12.52)) remained significantly associated with obesity. However, GORS was no longer significant.

\section{Medication use, BMI, and reflux}

Use of acid reducing drugs was a significant predictor for overall GORS (OR 9.8 (95\% CI 6.5, 14.7)) and for the following individual symptoms: heartburn (OR 6.4 (95\% CI $4.5,9.2)$ ), acid regurgitation (OR $6.2(95 \%$ CI $4.3,8.8)$ ), and retching (OR 3.0 (95\% CI 2.1, 4.2)). Drugs that potentially reduce LOS pressure (nitrates $(n=24)$, theophylline $(n=$ $10)$, calcium channel blockers $(n=44)$, opiates $(n=20)$, beta agonists $(n=22)$, phenothiazines $(n=2)$, tricyclic antidepressants $(n=2)$, nicotine substitutes $(n=0)$, anticholinergics $(n=0)$, and benzodiazepines $(n=2))$ as a group were univariately associated with the symptom of a burning feeling rising in the chest (Carlsson-Dent question) (OR 1.8 $(95 \%$ CI 1.1, 3.1)) and with central chest pain (OR $1.6(95 \%$ CI 1.0, 2.6)), but were not significantly associated with overall GORS. Only calcium channel blockers (OR 3.0 (95\% CI $1.5,5.9)$ ) were univariately associated with the symptom of a burning feeling rising in the chest; none of the other individual drug classes were significant. LOS relaxing drugs were not individually or as a group significantly associated with oesophagitis. Adjusting for medication use, the association between GORS and being overweight remained significant (OR 1.4 (95\% CI 1.04, 2.0)) and similarly, the association between GORS and obesity remained significant (OR 1.9 (95\% CI 1.3, 3.0)). The association between oesophagitis and BMI did not alter substantially adjusting for medication use (OR for overweight 1.7 (95\% CI 1.1, 2.6) and OR for obesity 3.4 (95\% CI $2.0,5.8)$ ). 
Table 4 Association of individual gastrointestinal symptoms with being overweight and obese based on body mass index (BMI) versus those of normal weight, among the study subjects $(n=973)$

\begin{tabular}{lll} 
& \multicolumn{1}{c}{ BMI 25-<30 } & BMI 30 \\
& OR (95\% CI) & OR (95\% CI) \\
\hline Weight loss & $0.31(0.11-0.89)$ & No cases \\
Anorexia & $1.23(0.57-2.65)$ & $0.56(0.15-2.04)$ \\
Uncomfortable feeling of fullness & $1.36(0.93-2.01)$ & $1.19(0.72-1.99)$ \\
Difficulty swallowing & $0.95(0.53-1.69)$ & $1.49(0.76-2.93)$ \\
Retching & $1.11(0.78-1.57)$ & $1.74(1.13-2.67)$ \\
Acid regurgitation & $1.33(0.95-1.86)$ & $2.30(1.52-3.48)$ \\
Early satiation & $1.32(0.86-2.03)$ & $1.0(0.55-1.79)$ \\
Nausea & $1.20(0.78-1.85)$ & $1.43(0.83-2.47)$ \\
Vomiting & $1.47(0.59-3.63)$ & $3.11(1.18-8.20)$ \\
Heartburn & $1.64(1.20-2.24)$ & $2.11(1.41-3.15)$ \\
Central chest pain & $1.17(0.83-1.67)$ & $1.38(0.88-2.16)$ \\
Burning feeling rising in chest & $1.51(1.03-2.23)$ & $1.99(1.24-3.21)$ \\
Constipation & $0.86(0.61-1.22)$ & $0.83(0.53-1.31)$ \\
Diarrhoea & $1.43(0.99-2.07)$ & $2.2(1.38-3.46)$ \\
Alternating constipation/diarrhoea & $1.14(0.73-1.76)$ & $1.25(0.72-2.18)$ \\
Feeling incomplete rectal evacuation & $1.16(0.84-1.60)$ & $1.64(1.09-2.47)$ \\
Pain at defecation & $0.96(0.60-1.52)$ & $0.88(0.47-1.67)$ \\
Pain relieved by defecation & $1.23(0.86-1.76)$ & $1.08(0.67-1.75)$ \\
Straining & $0.90(0.64-1.26)$ & $0.86(0.55-1.34)$ \\
Urgency & $1.05(0.74-1.49)$ & $1.60(1.04-2.47)$ \\
Flatus & $1.47(1.06-2.05)$ & $1.44(0.94-2.21)$ \\
Borborygmi & $1.23(0.90-1.69)$ & $0.97(0.63-1.50)$ \\
Abdominal distension & $1.08(0.79-1.47)$ & $0.98(0.64-1.48)$ \\
Nightly urge to defecate & $0.97(0.52-1.80)$ & $2.57(1.33-4.98)$ \\
Black stools & $1.21(0.43-3.42)$ & $0.37(0.05-3.07)$ \\
Blood in stool & $1.37(0.78-2.39)$ & $1.06(0.49-2.30)$ \\
Mucus & $0.61(0.36-1.04)$ & $1.16(0.64-2.12)$ \\
GORS & $1.53(1.14-2.06)$ & $2.05(1.39-3.01)$ \\
Epigastric pain & $0.96(0.67-1.39)$ & $1.63(1.05-2.55)$ \\
Dyspepsia & $1.00(0.74-1.36)$ & $1.42(0.96-2.11)$ \\
IBS & $1.21(0.88-1.66)$ & $1.58(1.05-2.38)$ \\
Abdominal pain & $1.19(0.89-1.58)$ & $1.59(1.08-2.35)$ \\
\hline Logistic regression adjusted for education, age and sex. & \\
OR (95\% CI), odds ratio (95\% confidence interval). & \\
BSS, irritable bowel syndrome; GORS, gastro-oesophageal reflux symptoms. & \\
& & \\
& & \\
& &
\end{tabular}

\section{DISCUSSION}

We have examined the associations between gastrointestinal symptoms and BMI in a population sample who were then investigated for an upper gastrointestinal tract structural explanation by oesophagogastroduodenoscopy. We found that reflux symptoms were linked to obesity and specifically, the presence of GORS was linked to reflux oesophagitis in the population. We also observed independent associations of obesity with diarrhoea-type symptoms.

We have confirmed the findings of other population based studies that showed an association between obesity and GORS. ${ }^{13} 1416{ }^{18} 36$ Lagergren et al did not find any association between obesity and GORS, but their definition of reflux was based on weekly reflux symptoms for a period of no less than one year and BMI data were obtained by self report. ${ }^{15}$ A study from the USA revealed an increased rate of reflux disease hospitalisation with higher BMI. ${ }^{37}$ We also observed a doseresponse effect, with the highest prevalence of GORS occurring in obesity. ${ }^{37}$ It has been speculated that a mechanistic role (from formation of a hiatal hernia) may be important in the genesis of these symptoms; on the other hand, an abnormal diet may not be important although strong data are not available. ${ }^{38}$ The striking result in the present study remains that the association between obesity and GORS was not evident when those with oesophagitis or peptic ulcer were excluded from the analyses. These data are consistent with the results from an earlier Swedish case control study. ${ }^{39}$

Lagergren et al have reported an association between medications that may relax the LOS and an increased risk for oesophageal adenocarcinoma. ${ }^{24}$ However, we failed to find any convincing association between these drugs as a group and GORS, although we did see an association between calcium channel blockers and the symptom of a burning feeling rising in the chest. Importantly, intake of medications did not substantially alter the association between BMI and GORS or BMI and oesophagitis in the multiple logistic regression models evaluated.

Obesity was not associated with constipation in this study; others have reported concordant observations. ${ }^{16-18}$ The finding of a link between obesity and diarrhoea, however, has now been confirmed in three population based studies, although these were all in uninvestigated subjects. ${ }^{16-18}$ Crowell et al also observed more frequent lower gastrointestinal symptoms in overweight females attending a weight management centre compared with normal weight women recruited from the community, ${ }^{40}$ although obese patients seeking treatment may not be representative of obese individuals in the community. Why does diarrhoea occur in obesity rather than, as might be expected in this generally more sedentary population, constipation? We hypothesise that excess intake of poorly absorbed products causing osmotic diarrhoea could explain the increased lower gastrointestinal symptoms in obesity. For example, there has been a very substantial increase in the use of corn syrup containing fructose in the USA, and excess ingestion of this could induce fructose malabsorption. ${ }^{41}{ }^{42}$ Other mechanisms that might explain the increased bowel frequency associated with increased BMI include abnormal bile salt turnover because of rapid small intestinal transit or rapid gastric emptying, which has been reported in some groups of obese patients. ${ }^{43}{ }^{44}$ Obesity was also associated with symptoms consistent with IBS in the present study. However, whether obesity is truly linked to IBS remains unclear; we did not apply the Rome II criteria for IBS as the questionnaire 
was not designed to assess these specifically. Others have observed a trend for more IBS symptoms in obesity but this has yet to be confirmed, and severe obesity has not been studied. ${ }^{45}$

Mechanisms that control food intake and energy expenditure may be dysregulated in obesity. A number of hormonal satiation factors, including cholecystokinin, enterostatin, and peptide YY from the gut, may contribute to meal termination, and thus may influence meal size. ${ }^{46}{ }^{47}$ Whether a decreased satiation response to food intake plays a role in the development of obesity is uncertain. ${ }^{48}{ }^{49}$ Early satiation, defined as an inability to finish a normal size meal, has been linked to impaired fundic accommodation in some studies although not all studies agree and the association is controversial.. ${ }^{51}$ For this reason, we investigated the association between obesity and the symptom early satiation; we speculated there would be more people with this symptom in those who were normal weight and less in the obese group. However, we did not observe increased reporting of early satiety in normal weight persons. This is contrary to previous observations in uninvestigated subjects with obesity ${ }^{16}{ }^{18}$; whether this reflects population or measurement differences is unknown.

Obesity is now considered to be a major health problem worldwide. Data from the National Center for Health Statistics show that $31 \%$ of the US population aged 20 years or above is clinically obese (BMI $\left.\geqslant 30 \mathrm{~kg} / \mathrm{m}^{2}\right){ }^{35}$ The prevalence of obesity in Northern Sweden was less (16\%) but still substantial; moreover, the rates of obesity in this cohort were only modestly higher than those reported across Sweden as a whole $(10 \%) .^{52}$ The present study had a number of other strengths. The ASQ is a reliable and adequately validated measure. ${ }^{25}{ }^{26}$ The study was performed in the northern part of Sweden, but the population studied appears to be representative of the Swedish population in terms of most sociodemographic factors, and the response rates were excellent. The proportion with higher education was slightly lower in these communities and a low education was associated with a higher BMI, but education was controlled for in the analyses. Hospitalisation and death from gastrointestinal disorders in the northern part of Sweden is similar to the rest of Sweden and the Western world. ${ }^{31}$ On the other hand, the impact of these gastrointestinal symptoms on quality of life in obese versus nonobese was not assessed in this study. However, we did ask only about troublesome symptoms, implying that the complaints reported were of importance to the community subjects.

In conclusion, in this population based study, reflux symptoms were independently associated with BMI. Importantly, the association was explained by increased upper endoscopy findings in obesity.

\section{ACKNOWLEDGEMENTS}

This study was supported in part by the Swedish Research Council, the Swedish Society of Medicine, Mag-tarm sjukas förbund, Norrbotten County Council, Sweden, and AstraZeneca R\&D, Sweden.

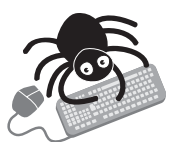

Conflict of interest: declared (the declaration can be viewed on the Gut website at http://www.gutinl.com/ supplemental)

\section{Authors' affiliations}

P Aro, J Ronkainen, T Storskrubb, E Bolling-Sternevald, L Agréus, Centre for Family Medicine, Karolinska Institiutet, Stockholm, Sweden N J Talley, Center for Enteric Neurosciences Translational and Epidemiological Research (CENTER), Mayo Clinic College of Medicine,
Rochester, MN, and Department of Medicine, University of Sydney and Nepean Hospital, Sydney, Australia

E Bolling-Sternevald, Astra Zeneca R\&D, Mölndal, Sweden

\section{REFERENCES}

1 Locke GR 3rd, Talley NJ, Fett SL, et al. revalence and clinical spectrum of gastroesophageal reflux: a population-based study in Olmsted County, Minnesota. Gastroenterology 1997;112:1448-56.

2 Nebel OT, Fornes MF, Castell DO. Symptomatic gastroesophageal reflux: incidence and precipitating factors. Am J Dig Dis 1976;21:953-56.

3 Jones RH, Lydeard SE, Hobbs FD, et al. Dyspepsia in England and Scotland. Gut 1990;31:401-5.

4 Talley NJ, Boyce $P$, Jones M. Identification of distinct upper and lower gastrointestinal symptom groupings in an urban population. Gut 1998;42:690-5.

5 Agreus L, Svardsudd K, Talley NJ, et al. Natural history of gastroesophageal reflux disease and functional abdominal disorders: A population-based study. Am J Gastroenterol 2001;96:2905-14.

6 Rajendra S, Kutty K, Karim N. Ethnic differences in the prevalence of endoscopic esophagitis and Barrett's esophagus: the long and short of it all. Dig Dis Sci 2004;49:237-42.

7 Fock KM, Talley NJ, Hunt RH, et al. Report of the Asia-Pacific consensus on the management of gastroesophageal reflux disease. J Gastroenterol Hepatol 2004; 19:357-67.

8 Hedley AA, Ogden CL, Johnson CL, et al. Prevalence of overweight and obesity among US children, adolescents and adults, 1999-2002. JAMA 2004;291:2847-50.

9 Stein CJ, Colditz GA. The epidemic of obesity. J Clin Endocrinol Metab 2004;89:2522-5.

10 Kawachi I. Physical and psychological consequences of weight gain. J Clin Psychol 1999;60(suppl 21):5-9.

11 White MA, O'Neil PM, Kolotkin RL, et al. Gender, race, and obesity-related quality of life at extreme levels of obesity. Obes Res 2004;12:949-55.

12 Kolotkin RL, Crosby RD, Williams GR. Health-related quality of life varies among obese subgroups. Obes Res 2002;10:748-56.

13 Locke GR 3rd, Talley NJ, Fett SL, et al. isk factors associated with symptoms of gastroesophageal reflux. Am J Med 1999;106:642-9.

14 Nilsson M, Johnsen R, Ye W, et al. Obesity and estrogen as risk factors for gastroesophageal reflux symptoms. JAMA 2003;290:66-72.

15 Lagergren J, Bergstrom R, Nyren $\mathrm{O}$. No relation between body mass and gastro-oesophageal reflux symptoms in a Swedish population based study. Gut 2000;47:26-9.

16 Talley NJ, Quan C, Jones MP, et al. The association of upper and lower gastrointestinal tract symptoms with body mass index in an Australian cohort. Neurogastro Motil 2004;16:413-19.

17 Talley N, Howell S, Poulton R. Obesity and chronic gastrointestinal tract symptoms in young adults: a birth cohort study. Am J Gastroenterol 2004;99:1807-14.

18 Delgado-Aros S, Locke GR III, Camilleri MC, et al. Obesity is associated with increased risk of gastrointestinal symptoms: A population-based study. Am J Gastroenterol 2004;99:1801-6.

19 Wong RK, Maydonovitch C, Garcia JE, et al. The effect of terbutaline sulfate nitroglycerine and aminofylline on lower esophageal sphincter pressure and radionuclide esophageal emptying in patients with achalasia. Clin Gastroenterol 1987;9:386-9.

20 Aggestrup S, Jensen SL. Effects of pirenzepine and atropine on basal lower esophageal pressure and gastric acid ecretion in man: a placebo controlled randomized study. Dig Dis 1991;9:360-4.

21 Gelfond M, Rozen P, Gilat T. Isosorbide dinitrate and nifedipine treatment of achalasia: a clinical, manometric and radionuclide avaluation. Gastroenterology 1982;83:963-9.

22 Ayres JG, Miles JF. Oesophageal reflux and asthma. Eur Respir J 1996;9:1073-8.

23 Rusnak MJ, Leevy CM. Effect of diazepam on the lower esophageal sphincter. A double-blind controlled study. Am J Gastroenterol 1980;73:127-30.

24 Lagergren J, Bergström R, Adami H-O, et al. Association between medications that relax the lower esophageal sphincter and risk for esophageal adenocarcinoma. Ann Intern Med 2000; 133:165-75.

25 Agreus L, Svardsudd K, Nyren O, et al. Irritable bowel syndrome and dyspepsia in the general population: Overlap and lack of stability over time. Gastroenterology 1995; 109:671-80.

26 Agreus L, Svardsudd K, Nyren O, et al. Reproducibility and validity of a postal questionnaire. The abdominal symptom study. Scand J Prim Health Care 1993; 11:252-62.

27 Drossman DA, Richter JE, Talley NJ. The functional gastrointestinal disorders. Boston: Little, Brown and Company, 1994

28 Agreus L, Talley NJ, Svardsudd K, et al. Identifying dyspepsia and irritable bowel syndrome: the value of pain or discomfort, and bowel habit descriptors. Scand J Gastroenterol 2000;35:142-51.

29 Carlsson R, Dent J, Bolling-Sternevald E, et al. The usefulness of a structured questionnaire in the assessment of symptomatic gastroesophageal reflux disease. Scand J Gastroenterol 1998;33:1023-9.

30 Klauser G, Schindbeck NE, Muller-Lissner SA. Symptoms in gastroesophageal disease. Lancet 1990;335:205-8.

31 ECE NBoHaW. Official database for 1998. Stockholm: National Board of Health and Welfare, 1998

32 Aro P, Ronkainen J, Storskrubb T, et al. Findings at upper endoscopy in a random adult population. Gastroenterology 2002;122(suppl 1):A568. 
33 Ronkainen J, Aro P, Storskrubb T, et al. High prevalence of gastroesophageal reflux symptoms and esophagitis with or without symptoms in the general adult Swedish population: a Kalixanda study report. Scand J Gastroenterol 2005;40:275-85.

34 Aro P, Ronkainen J, Storskrubb T, et al. Valid symptom reporting at upper endoscopy in a random sample of the Swedish adult general population: The Kalixanda study. Scand J Gastroenterol 2004;39:1280-8

35 Flegal KM, Carroll MD, Ogden CL, et al. Prevalence and trends in obesity among US adults, 1999-2000. JAMA 2002;288:1723-7.

36 Murray L, Johnston B, Lane A, et al. Relationship between body mass and gastro-oesophageal reflux symptoms: The Bristol Helicobacter Project. Int J Epidemiol 2003;32:645-50.

37 Ruhl CE, Everhart JE. Overweight, but not high dietary fat intake, increases risk of gastroesophageal reflux disease hospitalization: NHANES I Epidemiologic Followup Study. First National Health and Nutrition Examination Survey. Ann Epidemiol 1999;9:424-35.

38 Nandurkar S, Locke GR III, Fett SL, et al. Relationship between body mass index, diet, exercise and gastroesophageal reflux symptoms in a community. Aliment Pharmacol Ther 2004;20:497-505.

39 Nilsson $M$, Lundegårdh $G$, Carling $L$, et al. Body mass and reflux oesophagitis: an oestrogen dependent association? Scand J Gastroenterol 2002; 37:626-30.

40 Crowell MD, Cheskin U, Musial F. Prevalence of gastrointestinal symptoms in obese and normal weight binge eaters. Am J Gastroenterol 1994;89:387-91

41 Gross LS, Li L, Ford ES, et al. Increased consumption of refined carbohydrates and the epidemic of type 2 diabetes in the United States: an ecologic assessment. Am J Clin Nutr 2004;79:774-9.
42 Bray GA, Nielsen SJ, Popkin BM. Consumption of high-fructose corn syrup in beverages may play a role in the epidemic of obesity. Am J Clin Nutr 2004;79:537-43

43 Sadik R, Abrahamsson H, Ung KA, et al. Accelerated regional small bowel transit and overweight shown in idiopathic bile acid malabsorption. Am J Gastroenterol 2004;99:71 1-8.

44 Wisen 0, Hellstrom PM. Gastrointestinal motility in obesity. J Intern Med 1995;237:411-18

45 Locke GR III, Zinsmeister AR, Talley NJ, et al. Risk factors for irritable bowel syndrome: role of analgesics and food sensitivities. Am J Gastroenterol 2000;95:157-65.

46 Deutsch JA, Young WG, Kalogeris TJ. The stomach signals satiety. Science 1978;201:165-7

47 Geliebter A. Gastric distension and gastric capacity in relation to food intake in humans. Physiol Behav 1988:44:665-8.

48 Geliebter A, Westreich S, Gage D. Gastric distention by balloon and test-meal intake in obese and lean subjects. Am J Clin Nutr 1988;48:592-94.

49 French SJ, Murray B, Rumsey RD, et al. Preliminary studies on the gastrointestinal responses to fatty meals in obese people. Intl J Obes Rel Metab Disord 1993;17:295-300.

50 Camilleri M, Talley NJ. Pathophysiology as a basis for understanding symptom complexes and therapeutic targets. Neurogastroenterol Motil 2004; 16:135-42.

51 Feinle-Bisset C, Vozzo R, Horowitz M, et al. Diet, food intake, and disturbed physiology in the pathogenesis of symptoms in functional dyspepsia. Am J Gastroenterol 2004;99:170-81.

52 Sundquist K, Qvist J, Johansson SE, et al. Increasing trends of obesity in Sweden between 1996/97 and 2000/01. Intl J Obes Relat Metab Dis 2004;28:254-61.

\section{EDITOR'S QUIZ: GI SNAPSHOT}

\section{A case of jaundice with a mediastinal mass}

\section{Clinical presentation}

An elderly male presented with acute upper abdominal pain and tenderness with dyspnoea. On examination he had tachypnoea, tachycardia, hepatomegaly, and tenderness in the right upper quadrant and epigastrium. He also appeared jaundiced and had peripheral oedema of the lower limbs.

Laboratory findings were: aspartate transaminase $118 \mathrm{IU} / \mathrm{l}$ (normal range 0-32); alkaline phosphatase 430 IU/l (normal range 60-240); and bilirubin $40 \mu \mathrm{mol} / \mathrm{l}$ (normal range $<20$ ).

Chest radiograph performed at presentation suggested a mediastinal mass. Multidetector row computed tomography of the thorax and abdomen was performed for further assessment (fig 1A, B).

\section{Question}

What is the diagnosis?

See page 1390 for answer

This case is submitted by:

R Sinha

Department of Radiology, Glenfield Hospital, Leicester, UK

D Clarke

Department of Radiology, Derby City General Hospital, Derby, UK

Correspondence to: Dr R Sinha, Department of Radiology, Glenfield Hospital, Leicester LE3 9QP, UK; rakesh.sinha@uhl-tr.nhs.uk

doi: $10.1136 /$ gut.2005.066134

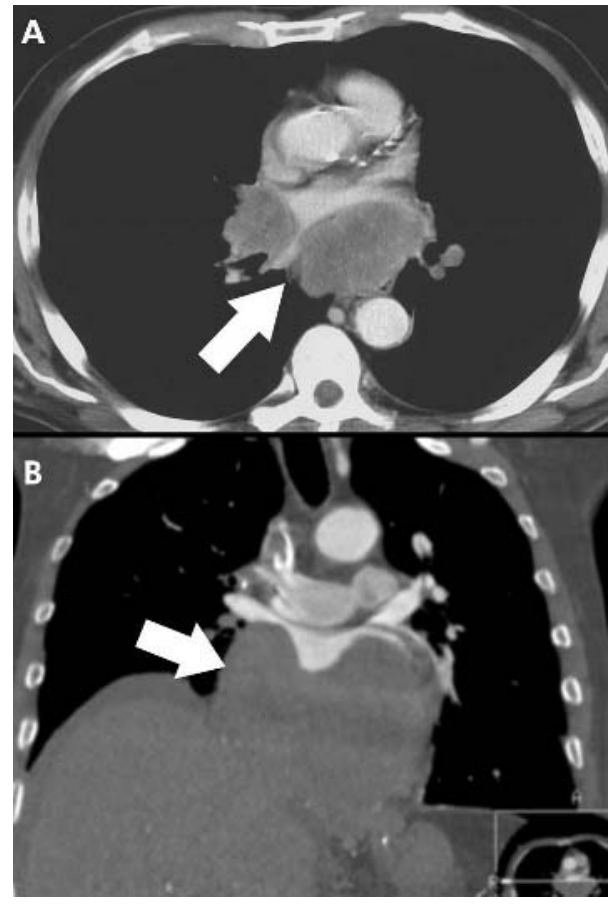

Figure 1 (A) Large lobulated mass involving the pericardium causing luminal compromise of the atria. (B) Coronal reformatted image along the plane of the right atrium and inferior vena cava shows large mass (arrow) with near total obliteration of the right atrial chamber. 